\title{
Title: ESTIMATES OF THE TEMPERATURE OF IMPACT CRATERS
}

For discussions outside the Laboratory

Date: March 1997
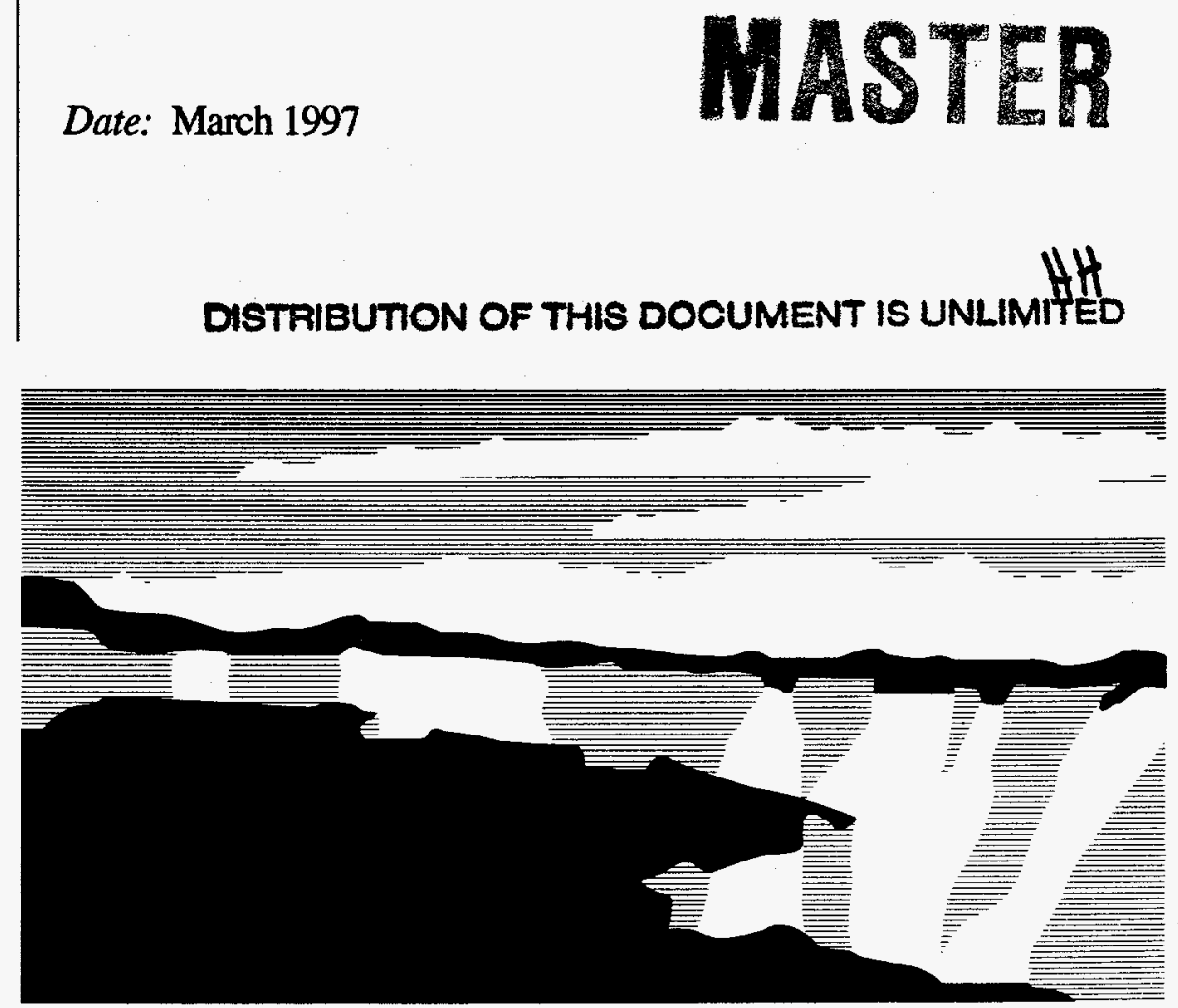

Los Alamos National Laboratory, an affirmative action/equal opportunity employer, is operated by the University of California for the U.S. Department of Energy under contract W-7405-ENG-36. By acceptance of this stricle, the publisher recognizes that the U.S. Government retains a nonexclusive, royalty-free license to publish or reproduce the published form of this contribution, or to allow others to do so, for U.S. Government purposes. The Los Alamos National Laboratory requests that the publisher identify this article as work performed under the auspices of the U.S. Department of Energy. 


\section{DISCLAIMER}

This report was prepared as an account of work sponsored by an agency of the United States Government. Neither the United States Government nor any agency thereof, nor any of their employees, make any warranty, express or implied, or assumes any legal liability or responsibility for the accuracy, completeness, or usefulness of any information, apparatus, product, or process disclosed, or represents that its use would not infringe privately owned rights. Reference herein to any specific commercial product, process, or service by trade name, trademark, manufacturer, or otherwise does not necessarily constitute or imply its endorsement, recommendation, or favoring by the United States Government or any agency thereof. The views and opinions of authors expressed herein do not necessarily state or reflect those of the United States Government or any agency thereof. 


\section{DISCLAMMIER}

Portions of this document may be illegible in electronic image products. Images are produced from the best available original document. 


\title{
ESTIMATES OF THE TEMPERATURE OF IMPACT CRATERS
}

\author{
Gregory H. Canavan
}

Scaling results for the growth and size of impact craters are used to estimate the temperatures and signals in impacts. The flow of plasma from the impactor entrance hole affords an opportunity to observe the thermodynamic state of the luminous material and infer the material properties of the target material. The brightness temperature can be determined from the optical thickness of the radiating layer, which can be solved using two-dimensional similarity solutions discussed earlier. The solutions are not overly sensitive to physical parameters. The peak signals should be readily observable from hundreds of kilometers.

Summary and conclusions. This note uses scaling results derived in companion notes for the expected growth ${ }^{1}$ and size $^{2}$ of impact craters to estimate the temperatures that should be observable in impacts. The impact plasmas will cool in milliseconds, but the flow of plasma out the impactor entrance hole affords an opportunity to observe the thermodynamic state of the luminous material and to infer the material properties of the target material. It is possible to develop scaling models of this interaction that are adequate for the formulation of estimates and assessment of sensitivities.

The quantity of principal interest is the brightness temperature, which can be determined from the optical thickness of the radiating layer. The resulting expression are solved both approximately and exactly for the temperature of the radiating layer. An essential feature of the solution is the knowledge from the two-dimensional similarity solutions discussed earlier that the plasma density at the exit plane is only a few percent of solid density, which closes the problem. The numerical integration produces temperatures somewhat higher than those from the analytic solutions for 1 and 10 microseconds, but the two estimates agree for 100 and 1,000 microseconds. The brightness temperatures fall from $1.2 \mathrm{ev}$ at 1 microsecond to $1 \mathrm{ev}$ at 10 microseconds; $0.75 \mathrm{ev}$ at 100 microseconds; and $0.6 \mathrm{ev}$ at 1,000 microseconds.

Only regions very close to the unit optical thickness surface contribute effectively to the radiation; thus, even large errors in the opacities, densities, or physical parameters used would only lead to a slight shift in the position and temperature of the radiating surface. The estimates of temperature as a function of time can be combined with the estimates of the exit hole size as a function of time from the previous paper to provide an estimate of the total observable signal. The peak signals should be readily observable from distances of hundreds of kilometers.

Background. The earlier note shows that the specific energy of the plasma produced by the impact of an object at speed $V$ is on the order of $V^{2} / 2$. For impact velocities on the order of $10 \mathrm{~km} / \mathrm{s}$, this implies initial debris temperatures on the order of $1-2 \mathrm{ev}, 3$ although those temperatures fall on the time scale of hydrodynamic expansion, which is about $30 \mathrm{~cm} / 100 \mathrm{~m} / \mathrm{s}$ 
sound speed $\approx 300$ microseconds. During that time, the impact debris both expands into the surrounding material and flows out the impactor entrance hole. The former is treated in the previous note. The latter affords an opportunity to observe the thermodynamic state of the luminous material and to infer from it the material properties of the target material.

The treatment of impact process is complicated by the irregular geometry of the impactor and target, the unknown shape and composition of the target, and the unknown equation of state and preexisting fractures in the target. However, it is possible to make scaling models of the interaction adequate for the formulation of estimates and the assessment of sensitivities.

Impact. The simplest model estimates the brightness temperature of the material in the impactor entrance hole from the scaling model used in the previous paper. The similarity solutions of the partial differential equations for the two-dimensional impact problem are approximate, but produce several robust predictions. The most important is that the flow out the entrance hole is largely the isentropic expansion that results from unloading behind the shock.

The second is that in very high-speed impacts, while the entrance hole at the top of the roughly spherical bowl-shaped cavity is large, only about 1.6 percent of the mass escapes through it, because the density in the hole is only $\approx 2 \%$ of the density of the solid target material.

The density of the material at the bottom of the bowl is $\approx 10$ times solid density, about the value expected from a strong shock. Thus, there is a fairly rapid 1000 -fold expansion across the cavity followed by a much slower expansion outside the entrance. These results vary only slowly with gas dynamic parameters, impactor material, and geometry. Of particular importance is the result that the energy loss out of the hole is not large, so that the observable phenomena can be treated as a small loss to first order accuracy.

Model. The similarity solutions for the flow from the entrance hole can solve for opacity and temperature of the exterior flow field. The quantity of principal interest is the brightness temperature of the flow, which is the effective temperature at which the radiant energy is emitted. The optical thickness of the radiating layer at distance $x$ can be written as

$$
\tau(x)=\int_{0} x^{\prime} x^{\prime} K\left(x^{\prime}\right)
$$

where $\mathrm{K}(\mathrm{x})$ is the frequency averaged absorption coefficient at $\mathrm{x}$. In the rarefaction flow field, the temperature $T$ is uniquely related to $x$, so Eq. (1) can also be written as

$$
\tau\left(T_{b r}\right)=-\int_{0} T b r d T K(T) d x / d T=1,
$$

where by the definition above, the optical thickness $\tau\left(\mathrm{T}_{\mathrm{br}}\right)$ at $\mathrm{T}_{\mathrm{br}}$ is unity, since the optical thickness unity surface is where the radiation originates. The Jacobian $\mathrm{dx} / \mathrm{dT}$ can be evaluated from the similarity solution for the rarefaction as 4

$$
\mathrm{dx} / \mathrm{dT} \approx-[(\gamma+1) \mathrm{ct}] /[2(\gamma-1) \mathrm{T}]
$$

where $\gamma$ is the ratio of specific heats, which is $\approx 1.2$ in this temperature region, and $t$ is the scaling time since impact. In the region where the primary absorption mechanism is photoelectric 
absorption by highly excited atoms and bremsstrahlung on ions, the absorption coefficient can be approximated by the Kramers Unsold formula 5

$$
\mathrm{K}=\mathrm{ANe}-\mathrm{E} / \mathrm{kT} / \mathrm{T}^{2} \text {, }
$$

where $\mathrm{N}$ is the density, $\mathrm{E}$ is the effective ionization energy, and $\mathrm{A}$ is a constant $\approx 3 \times 10^{-17} \mathrm{~cm}^{2}$. $\mathrm{ev}^{2}$, which depends on the inverse of the cube of the photon frequency. The effective ionization energy is $E=I-h v$ for photo ionization. For weakly ionized plasmas with Saha like ionization, absorption is by bremsstrahlung in the field of neutrals, so that

$$
\mathrm{K} \sim \mathrm{NN}_{\mathrm{e}} \sim \mathrm{Be}^{-\mathrm{I} / 2 \mathrm{kT} / \mathrm{N}^{3 / 2}} \text {. }
$$

While the coefficients are different in Eqs. (4) and (5), the dominant dependence is their exponential dependence on $T$. Since for the photons of interest, $\mathrm{I}-\mathrm{hv} \approx \mathrm{I} / 2$, the two equations give similar absorptions to within the approximations implicit in either. Thus, Eq. (4) with I = 5 ev is used below to produce typical estimates. Substituting Eqs. (3) and (4) into Eq. (2) gives

$$
\int_{0} \mathrm{Tbr}_{\mathrm{dT} \mathrm{ANe}}-\mathrm{E} / \mathrm{kT} / \mathrm{T}^{2}[(\gamma+1) \mathrm{ct}] /[2(\gamma-1) \mathrm{T}]=1 \text {. }
$$

The dominant dependence in this expression is that of $\mathrm{e}^{-\mathrm{E} / \mathrm{kT}}$ on $\mathrm{T}$. If other dependencies are suppressed, that produces 6

$$
\begin{aligned}
& \mathrm{AN}[(\gamma+1) \mathrm{ct}] /[2(\gamma-1)] \int_{\mathrm{o}} \mathrm{Tbr}_{\mathrm{dTe}}-\mathrm{E} / \mathrm{kT}_{\mathrm{T}} / \mathrm{T}^{3} \approx \mathrm{AN}[(\gamma+1) \mathrm{ct}] /\left[2(\gamma-1) \mathrm{T}^{2}\right] \mathrm{e}-\mathrm{E} / \mathrm{kTbr}=1 \\
& \mathrm{~T}_{\mathrm{br}} \approx \mathrm{E} / \mathrm{k} \ln \left\{\mathrm{AN}[(\gamma+1) \mathrm{ct}] /\left[2(\gamma-1) \mathrm{T}^{2}\right]\right\}
\end{aligned}
$$

which is shown in Fig. 1 . The top curve is for an exit plane density of $0.1 \%$ of solid; the bottom for $1 \%$ of solid density. As noted above, two-dimensional similarity solutions for the impact bowl indicate that the density at the exit plane is about $2 \%$ of solid density, so these values roughly bound the expected variations. The calculations cover from the time of impact to about 100 microseconds. For longer times, the expansion would become strongly three dimensional. That would violate the formal assumptions in the solutions, although it should not impact the trends seen, since the three dimensional effects would only be felt at large distances, which contribute little to absorptions.

The top curve gives a brightness temperature of about $1 \mathrm{ev}$ for times shorter than 1 microsecond. By 10 microsecond the brightness temperature has fallen to about $0.55 \mathrm{ev}$. By 100 microsecond it has fallen to about $0.45 \mathrm{ev}$. The lower curve is for a density $1 \%$ of solid, which gives a lower temperature throughout because of the factor of $\mathrm{N}$ in the denominator of Eq. (8). The origin of that factor is the $\mathrm{N}$ in Eq. (4), which indicates that at higher density, a given absorption-and hence optical depth-can be produced at a lower temperature. On the higher density curve, the temperature at 1 microsecond is about $0.55 \mathrm{ev}$. It falls to 0.45 and 0.35 at 10 and 100 microseconds. The fall in brightness temperature is only logarithmic in Eq. (8), reflecting the fact that it only enters through the slow reduction of the density in the expansion.

Optical depth. Performing the integrals in Eq. (6) numerically provides some additional insight into the structure of the radiating region. Figure 2 shows the optical depth as a function of 
temperature, which can be converted with the similarity solution to optical depth as a function of distance, if needed. The curves are for a density intermediate between the two values used on Fig. 1. Figure 1 concentrates on the region around unit optical depth. In Fig. 2, at 1 microsecond, that corresponds to a temperature of $1.2 \mathrm{ev}$. At 10 microseconds, it corresponds to a temperature of $1 \mathrm{ev}$. At 100 microseconds, it corresponds to a temperature of $0.75 \mathrm{ev}$. At 1,000 microseconds, it corresponds to a temperature of $0.6 \mathrm{ev}$.

The brightness temperatures from the numerical integration produce temperatures that are somewhat higher than those from the analytic solutions for 1 and 10 microseconds, but the two estimates largely agree for 100 and 1,000 microseconds. Figure two shows that the temperature well into the cavity could be on the order of $2 \mathrm{ev}$, but those regions are separated from the radiating surface by an optical thickness of up to $10^{4}$. Radiation transport is essentially local in optically thick regions, so essentially none of the higher energy photons emitted in the high temperature region survive to contribute to the exiting flux.

At $10 \mathrm{~ns}$ even near the radiating surface, the regions with temperatures $10 \%$ above and below the $1 \mathrm{ev}$ radiating region would be strongly isolated from it. The $1.1 \mathrm{ev}$ surface would be isolated from it by an optical thickness of 3-4. The $0.9 \mathrm{ev}$ surface would only have an optical thickness of 0.4 ; hence, it would only have about $40 \%$ of the radiation density of a black body and would radiate weakly. The principal result is that only regions very close to the unit optical thickness surface contribute, as assumed in the analytic solution of Fig. 1. The converse observation is that even a large error in the opacities, densities, or physical parameters used would only lead to a slight shift in the position and temperature of the radiating surface.

Signal. The estimates of temperature as a function of time can be combined with the estimates of the exit hole size as a function of time from the previous paper to provide an estimate of the total observable signal, which is shown in Fig. 3. At 1 microsecond the signal is about $7 \mathrm{MW} / \mathrm{sr}$. It falls due to the fall in brightness temperature in Fig. 2 . The decrease is mitigated slightly by the $\approx 4$-fold increase in hole area by about 100 microsecond, as estimated with the radius $\alpha \mathrm{t}^{2 / 5}$ scaling law of the previous note. ${ }^{7}$ Beyond that time the fall is more rapid, reaching about $2 \mathrm{MW} / \mathrm{sr}$ by 1 millisecond. It would appear that these signals should be readily observable from distances of hundreds of kilometers.

Summary and conclusions. This note uses its scaling results derived earlier for the expected growth and size of impact craters to estimate the temperatures that should be observable in impacts. The earlier notes estimated that for impact velocities on the order of $10 \mathrm{~km} / \mathrm{s}$, initial debris temperatures should be on the order of 1-2 ev. They will cool in milliseconds, but the flow of debris out the impactor entrance hole affords an opportunity to observe the thermodynamic state of the luminous material and to infer the material properties of the target material. Despite 
uncertainties, it is possible to develop scaling models of the interaction adequate for the formulation of estimates and assessment of sensitivities.

The quantity of principal interest is the brightness temperature, which can be determined from the optical thickness of the radiating layer. The resulting expression can be solved either approximately or exactly for the temperature of the radiating layer. An essential feature of the solution is the knowledge from the two-dimensional similarity solutions discussed earlier that the plasma density at the exit plane is only a few percent of solid density, which closes the problem. The numerical integration produces temperatures that are somewhat higher than those from the analytic solutions for 1 and 10 microseconds, but the two estimates largely agree for 100 and 1,000 microseconds. The brightness temperatures fall from $1.2 \mathrm{ev}$ at $1 \mathrm{microsecond}$ to $1 \mathrm{ev}$ at 10 microseconds; $0.75 \mathrm{ev}$ at 100 microseconds; and $0.6 \mathrm{ev}$ at 1,000 microseconds.

Only regions very close to the unit optical thickness surface contribute effectively to the radiation, as assumed in the analytic solutions in the literature. Conversely, even large errors in the opacities, densities, or physical parameters used would only lead to a slight shift in the position and temperature of the radiating surface. The estimates of temperature as a function of time can be combined with the estimates of the exit hole size as a function of time from the previous paper to provide an estimate of the total observable signal. The peak signal at about 1 microsecond is about $7 \mathrm{MW} / \mathrm{sr}$, falling to about $2 \mathrm{MW} / \mathrm{sr}$ by 1 millisecond, which should be readily observable from distances of hundreds of kilometers.

\section{References}

1. G. Canavan, "Mass Ejected by Impacts with Materials of Various Strengths," Los Alamos National Laboratory report LA-UR-97-13, January 1997.

2. G. Canavan; Crater Scaling on Projectile Size and Speed," Los Alamos National Laboratory report LA-UR-97-929, March 1997.

3. Ya. B. Zel'dovich and Yu. P. Raizer, Physics of Shock Waves and High-Temperature Hydrodynamic Phenomena (Academic Press, New York and London, 1967), Vol. I p. 212, Table 3.5.

4. Ya. B. Zel'dovich and Yu. P. Raizer, Physics of Shock Waves and High-Temperature Hydrodynamic Phenomena, op. cit., p. 775, Eq. (11.72).

5. Ya. B. Zel'dovich and Yu. P. Raizer, Physics of Shock Waves and High-Temperature Hydrodynamic Phenomena, op. cit., p. 271, Eq. (5.44).

6. Ya. B. Zel'dovich and Yu. P. Raizer, Physics of Shock Waves and High-Temperature Hydrodynamic Phenomena, op. cit., p. 777.

7. G. Canavan, "Mass Ejected by Impacts with Materials of Various Strengths," op. cit. 


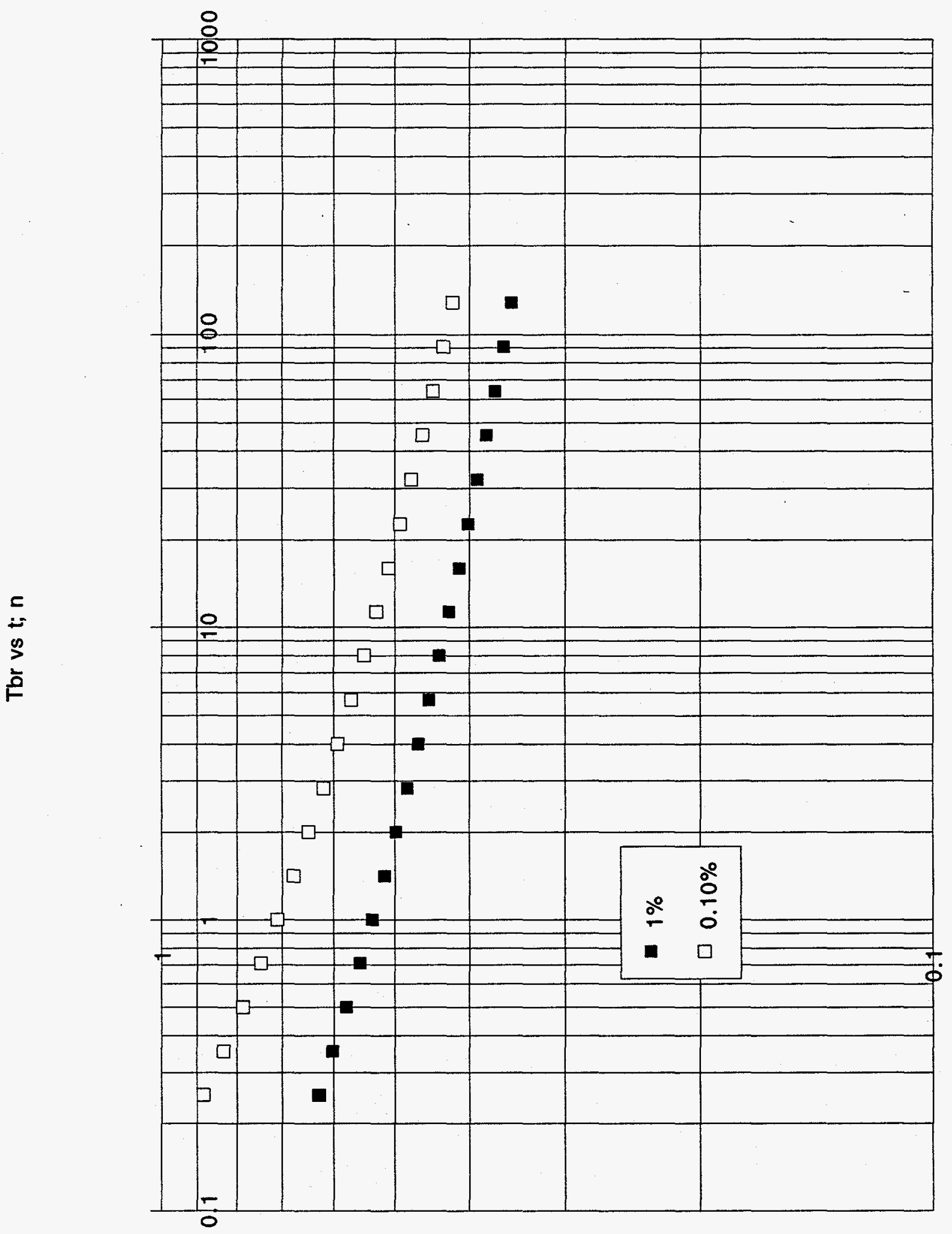

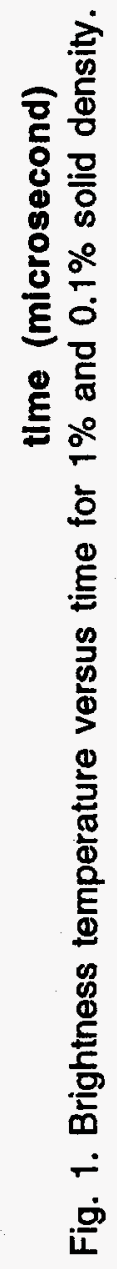

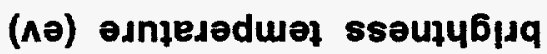




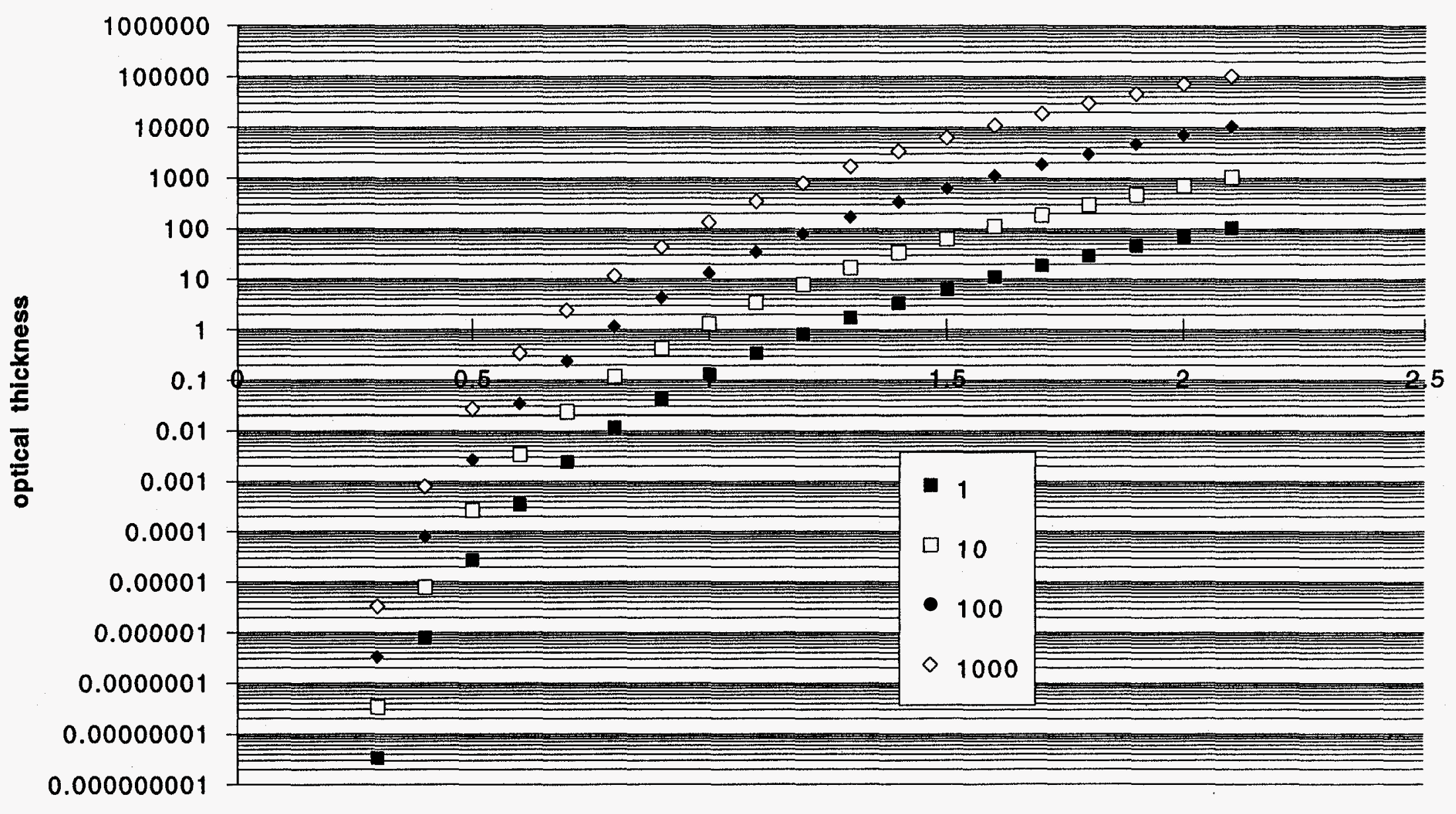

\section{temperature (ev)}

Fig. 2. Optical thickness versus temperature at various times (microsecond). 


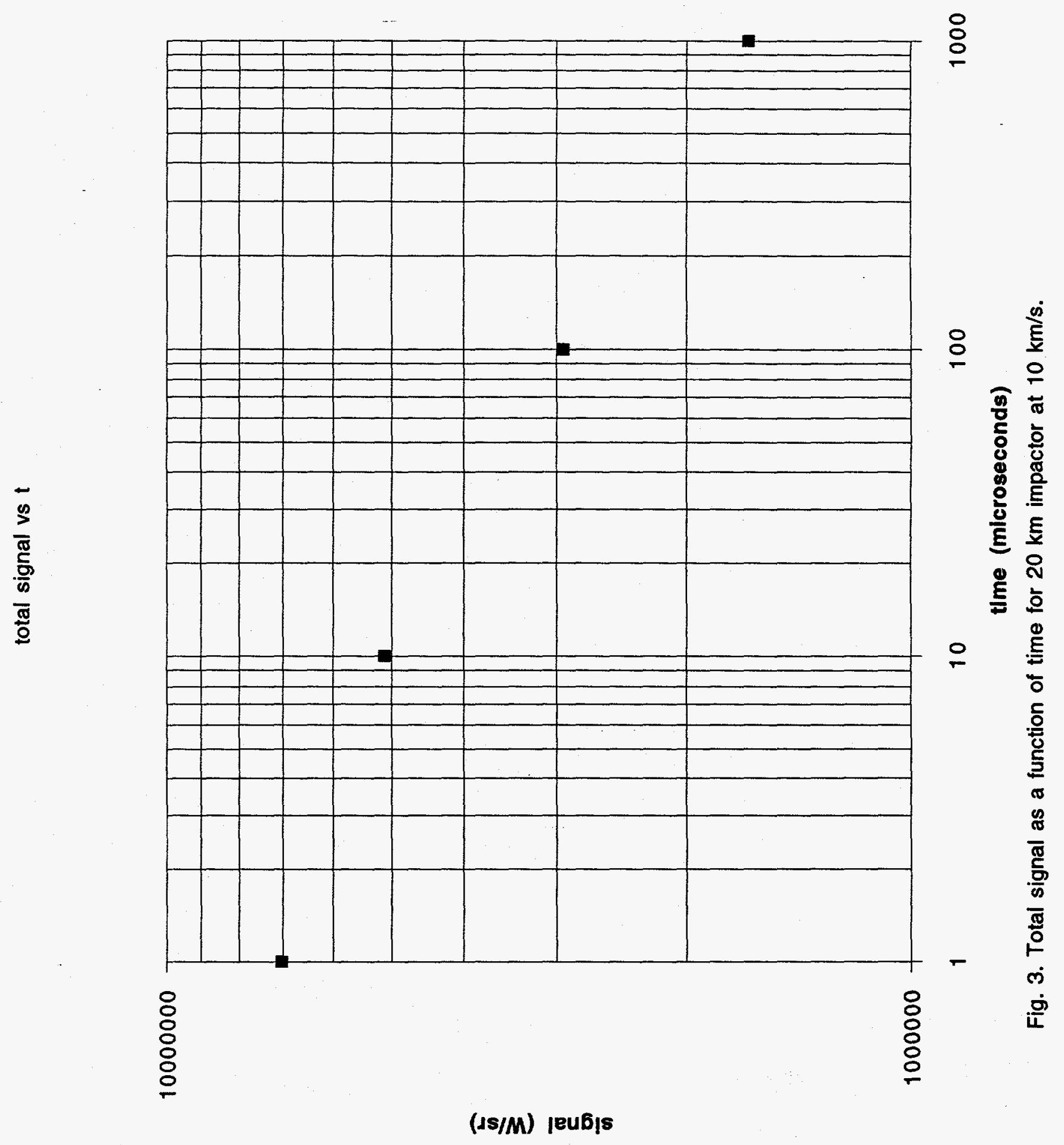

